\title{
Pérdidas causadas por el nematodo Quiste de la papa (Globodera sp.) en Bolivia y Penú
}

\section{J. Franco-Ponce1; A. González-Verástegui² \\ Resumen}

Las pérdidas económicas causadas por Globodera sp. en Bolivia y Perú se estimaron en base a información documentada disponible sobre su distribución (incidencia) y pérdidas en el rendimiento (severidad) del cultivo de papa. Se determinó que Globodera sp. está presente en diferentes niveles de infestación en todos los departamentos andinos de ambos países. La extrapolación de áreas cultivadas, incidencia, severidad de daño y precio de venta de los tubérculos, mostraron que las pérdidas económicas en el valor bruto de la producción de papa en Bolivia y Perú alcanzan a US\$ 13'000,000 y US\$ $128^{\prime} 000,000$, respectivamente. La especie dominante en el Perú es G. pallida y G. rostochiensis en Bolivia, aun cuando en este último se encontraron poblaciones con ambas especies. Globodera $s p$. fue más frecuente entre los 3,500 y 4,000 msnm.

Palabras claves adicionales:

Incidencia, severidad, pérdidas económicas.

Aceptado para publicación: Diciembre 28, 2011

${ }^{1}$ Consultor, Fundación PROINPA, Casilla Postal 4285, Cochabamba, Bolivia i.franco@proinpa.org

${ }^{2}$ ONG DESOAGRO, Lima, Perú. 


\section{Losses caused by potato cyst nematode (Globodera sp.) in Bolivia and Penu}

\section{Summary}

Economic losses caused by Globodera sp. in Bolivia and Peru were estimated on basis of available documented information concerning its distribution (incidence) and yield losses (severity) in potato crop. It was established that Globodera sp. is present at different infestation densities in all prospected Andean departments of both counties. Extrapolation of cultivated areas, incidence, severity of damage, and price of the tubers, showed an economic loss of the gross value of potato production in Bolivia and Peru of US $\$ 13,000.000$ and US $\$ 128{ }^{`} 000,000$, respectively. The dominating species in Peru was G. pallida and $G$. rostochiensis in Bolivia, although mixed populations of both species were also found. Globodera species were most frequently found between 3,500 masl and 4,000 masl.

Additional key words:

Incidence, severity, gross value losses.

\section{Introducción}

Los territorios de Perú y Bolivia en la actualidad se encuentran ubicados en lo que es la región altiplánica que circunda el lago Titicaca que representa el principal centro de origen de la papa cuya antigüedad data de 7,000 años antes de las culturas preincas e incas; y a la vez es el centro de mayor biodiversidad de papas cultivadas de diversas ploidías (3400 entradas en 8 grupos de especies y 2 sub-especies) y de 199 especies silvestres (Huaman y Spooner, 2002; Hijmans y Spooner, 2001). El cultivo de la papa se realiza bajo un amplio rango de altitudes, latitudes y condiciones climáticas que supera a cualquier otro cultivo de importancia económica mundial. Sin embargo, las principales limitantes del cultivo de papa se presentan en áreas geográficas específicas en los países de Latino América. 
Los rendimientos de Bolivia (5.7 t/ha) y Perú (16.8 t/ha) están por debajo del promedio mundial (17.6 t/ha) y muy lejos de los países líderes como Nueva Zelandia (45.7 t/ha), Bélgica (43.9 t/ha) o Países Bajos (43.2 t/ha). Existen muchas razones agronómicas, climáticas, socio-económicas e institucionales que explican tales diferencias.

En el Perú, la Sierra concentra el $68 \%$ de la superficie y $87 \%$ de la producción nacional, liderada por Puno y Cusco en el Sur (donde se cultiva entre Septiembre y Mayo), Huánuco y Junín en el Centro y La Libertad y Cajamarca en el Norte, donde se cultiva buena parte del año. El rendimiento nacional en el Perú muestra diferencias muy fuertes entre la Sierra (7 a $20 \mathrm{t} / \mathrm{ha}$ ), con condiciones muy heterogéneas de cultivo y altos riesgos, y la Costa (25 a $30 \mathrm{t} / \mathrm{ha}$ y hasta $40 \mathrm{t} / \mathrm{ha}$ ), donde se cultiva en zonas más accesibles, con agua de riego y un nivel de riesgo mucho menor. En Bolivia, el 93\% de la producción primaria se hace en tres de las cinco eco-regiones (Puna con el 50\%, Puna Alta: $23 \%$ y Valles: 20\%) y está repartida entre los departamentos de La Paz, Potosí, Cochabamba, Oruro y Tarija. La mayor parte se cultiva en época de siembra grande, siembra temprana o medio temprana, en condiciones de suelos pobres y alto riesgo climático.

La mayor parte de la papa producida se consume sin procesar (90\% o más), razón por la cual la papa fresca sigue siendo un alimento básico para la gran mayoría de la población pobre, particularmente en las zonas rurales andinas donde no existe infraestructura adecuada para almacenarla ni procesarla. El consumo per cápita en fresco para el periodo 2002-2006 estaba en 43.3 y 68.4 kg/año en Bolivia y Perú, respectivamente, superando al promedio mundial (36.5 kg/año). El rubro papa contribuye con $11.0 \%$ y $10.0 \%$ del PBI agrícola en Perú y Bolivia, respectivamente (Devaux et al., 2010).

Sin duda alguna, entre los problemas fitosanitarios más importantes en estos países andinos están los nematodos fitoparásitos que limitan su producción en la zona andina temperada y que han sido más estudiados, se encuentra el nematodo quiste de la papa (Globodera rostochiensis y $G$. 
pallida). Estos nematodos, desde que la papa se cultivó en la región andina desde por lo menos hace 8000 anos AC, han coevolucionado con su hospedante el género Solanum sp. (Franco, 1977 ; 1994).

El nematodo quiste de la papa fue reportado por primera vez en el Perú en 1952 (Willie y Bazan de Segura, 1952). A partir de esa fecha su distribución en la región Andina de Perú y la presencia de ambas especies (G. pallida Stone G. rostochiensis Wollenweber) con diferentes razas fu confirmado y establecido (Evans et al., 1975). En Bolivia el primer reporte de la presencia de Globodera spp. fue el año 1955 ( Bell et al., 1955).G. pallida ha mostrado una distribución más amplia que G. rostochiensis en la región Andina de América del Sur, y G. pallida es también en la actualidad la especie más común en la mayoría de las áreas paperas del mundo (Franco, 1994).

Los factores que soportan la importancia de estos nematodos en la producción de papa, son por un lado su distribución, la existencia de razas, los medios de diseminación, y por otro lado, la no disponibilidad de medidas efectivas de control y de aceptación por parte de los productores, tales como cultivos alternantes rentables, variedades resistentes, semilla certificada, fertilizantes orgánicos, y otras medidas fitosanitarias de orden legal que impidan su diseminación. Por otro lado no se cuenta con información sobre las pérdidas que estos nematodos causan en el cultivo de papa en los países latinoamericanos donde están presentes. En esta oportunidad se presenta uno de los estudios más completos que se han efectuado sobre las pérdidas económicas causadas por estos nematodos en Bolivia y Perú.

\section{Materiales y Métodos}

Inicialmente se colectó toda la información disponible sobre la incidencia (presencia o ausencia en lugares de muestra: frecuencia) y la severidad (escalas pre-determinadas de acuerdo a los niveles de infestación: intensidad) por Globodera $\mathrm{sp}$ y las pérdidas que éstos causan en el rendimiento de papa en las regiones paperas de ambos países. La mayor parte de la 
información colectada de numerosas tesis de grado y estudios de diagnóstico efectuados, fue ordenada por departamento de acuerdo a la presencia o ausencia del nematodo. Con esta información, se calculó el porcentaje de incidencia de Globodera sp., a través de una fórmula que consideraba el número de parcelas afectadas sobre el número total de parcelas muestreadas. La severidad de daño por Globodera sp., es un índice que se relaciona con las pérdidas de rendimientos en el cultivo de papa, que en algunos casos se establece a través del número de quistes en raíces directamente extraídas del campo y de acuerdo a una escala que considera los niveles o grados de infestación del suelo, y en la mayoría de los casos se establece por el número de quistes por 100 gramos de suelo En otros casos se determina la severidad de Globodera sp. por la infestación del suelo en número de huevos y/o estados juveniles por gramo de suelo, que en todo caso es la más recomendable pero requiere de cierto equipo y de personal entrenado. Fue esta última la que se consideró en el presente estudio para estimar las pérdidas de rendimiento (Franco y González, 1993; González y Franco, 1993; Franco et al., 1998/1999).

Es así que las pérdidas de rendimiento, que corresponden a los rendimientos que se dejan de obtener, se establecieron por la fórmula que se describe a continuación y que utilizó toda la información obtenida sobre la incidencia y el grado de severidad de daño por Globodera sp., de acuerdo a escalas de pérdidas o de reducción de los rendimientos previamente establecidas (Tabla 1) y de los rendimientos promedio por departamento (Tabla 2 y 3 ).

Daño o Pérdida en rendimiento $(\%)=$ (Incidencia $(\%) \times$ Severidad (Escala \% reducción)) / 100

Toda la información obtenida de los departamentos Andinos de Bolivia y Perú, fueron tabulados, analizados y extrapolados con las áreas cutivadas de papa en relación a la incidencia, severidad de daño y precio de venta de los tubérculos, permitieron estimar las pérdidas económicas en el valor bruto de la producción de papa en Bolivia y Perú. 
Tabla 1. Niveles de infestación de los suelos por Globodera sp. y los porcentajes de pérdidas o de reducción en el rendimiento, en base al número de huevos y $\mathrm{J} 2$ por gramo de suelo

\begin{tabular}{|l|c|c|}
\hline $\begin{array}{c}\text { Grado infestación } \\
\text { suelo }\end{array}$ & $\begin{array}{c}\text { Huevos + J2/ gramo } \\
\text { suelo }\end{array}$ & $\begin{array}{c}\text { Pérdidas de } \\
\text { rendimiento (\%) }\end{array}$ \\
\hline Libre & 0 & 0 \\
\hline Incipiente & $1-5$ & 5 \\
\hline Media & $5.1-15$ & 13 \\
\hline Alta & $15.1-35$ & 45 \\
\hline Muy Alta & $>35$ & 58 \\
\hline
\end{tabular}

Fuente: Lanza, 1996; Tola, 1997; Peralta, 1995; Maín, 1994; Esprella, 1993.

Tabla 2. Rendimientos promedio departamentales a nivel agricultor y áreas cultivadas de papa en Bolivia

\begin{tabular}{|l|c|c|}
\hline \multicolumn{1}{|c|}{ Departamentos } & $\begin{array}{c}\text { Área Cultivada } \\
\text { (has } \mathbf{x} \text { 000) }\end{array}$ & $\begin{array}{c}\text { Rdto promedio } \\
\text { (t /ha) }\end{array}$ \\
\hline Cochabamba & 25,9 & 6,76 \\
\hline Potosí & 30,8 & 5,05 \\
\hline La Paz & 37,8 & 5,08 \\
\hline Tarija & 7,5 & 6,94 \\
\hline Chuquisaca & 19,8 & 5,75 \\
\hline Oruro & 9,7 & 3,79 \\
\hline & Total:131, 3 has & $-\mathbf{x : ~ 5 , ~ 5 3 ~ t / h a ~}$ \\
\hline
\end{tabular}

Fuente: Zeballos, 1997; INE, 1994. 
Tabla 3. Rendimientos promedio departamentales a nivel agricultor y áreas cultivadas de papa en Perú

\begin{tabular}{|l|c|c|}
\hline \multicolumn{1}{|c|}{ Departamentos } & Area cultivada & Rendimiento \\
\hline Cajamarca & 25,1 & 9,07 \\
\hline La Libertad & 20,8 & 14,99 \\
\hline Junín & 22,5 & 14,39 \\
\hline Ayacucho & 14,2 & 11,45 \\
\hline Huancavelica & 14,8 & 9,15 \\
\hline Huánuco & 26,3 & 13,33 \\
\hline Apurímac & 25,5 & 10,10 \\
\hline Arequipa & 6,1 & 23,64 \\
\hline Cuzco & 27,1 & 9,54 \\
\hline Puno & 37,1 & 8,36 \\
\hline & Total: $\mathbf{2 1 9 , 5}$ has & $-\mathbf{x :} \mathbf{1 1 , 4 1}$ t/ha \\
\hline
\end{tabular}

Fuente: MINAG 2007

\section{Resultados y Disc usión}

Los departamentos muestreados en ambos países (5 en Bolivia y 9 en Perú) mostraron la presencia del nematodo quiste de la papa, Globodera sp. En Perú de 3299 muestras de suelo, 64.90 $\%$ fueron positivas y el nivel de infestación alcanzó hasta 2161 huevos/gramo de suelo (Tabla 4).

En el Perú la mayor incidencia de muestras positivas se presentó en los departamentos de Cusco, Huánuco, La Libertad y Huancavelica $(90.45,84.11,80.06,78.22 \%$, respectivamente). Por otro lado, Huancavelica y Junín (55.44 y $41.67 \%$ ) mostraron los más altos niveles de severidad, con niveles de infestación alta y muy alta (15.1-35 y >35 huevos+j2 / gramo de suelo). Los 
departamentos que aun mostraron áreas libres fueron Cajamarca y Ayacucho (69.04 y 62.08 \%) Los otros departamentos mostraron niveles de infestación incipiente y media (1-5 Huevos/g suelo y 5.1-15 Huevos/g suelo). Los mayores niveles de infestación se tuvieron en los departamentos de Junín, Huancavelica, Apurimac, Cusco y Puno (2161, 2130, 1329, 840 y 821 Huevos/g suelo, respectivamente). Las pérdidas en rendimiento estimadas en los departamentos andinos variaron de acuerdo a los diversos niveles de infestación establecidos para cada uno de ellos. Las menores pérdidas fueron para los departamentos de Cajamarca, Ayacucho y Arequipa, no así para los departamentos de Cusco, Junín, Huánuco y La Libertad que mostraron las mayores pérdidas (Figura 1).

Tabla 4. Niveles de infestación por Globodera sp. (Huevos +Juveniles 2) en muestras de suelo de diferentes departamentos andinos del Perú

\begin{tabular}{|l|c|c|}
\hline \multicolumn{1}{|c|}{ Departamentos } & \multicolumn{2}{c|}{ Huevos+J2/cc. suelo } \\
\hline & Rango & Promedio \\
\hline Cajamarca & $1,00-641,56$ & 205,36 \\
\hline La Libertad & $1,00-93,51$ & 28,82 \\
\hline Junín & $1,02-2161,38$ & 466,86 \\
\hline Ayacucho & $1,00-508,90$ & 121,32 \\
\hline Huancavelica & $1,80-2130,00$ & 1065,90 \\
\hline Huánuco & $1,05-290,58$ & 107,18 \\
\hline Apurímac & $1,00-1328,80$ & $664, .90$ \\
\hline Arequipa & $1,09-647,52$ & $156 ., 33$ \\
\hline Cuzco & $14,00-840,00$ & 254,51 \\
\hline Puno & $0,00-820,76$ & 92,88 \\
\hline & $\mathbf{0 , 0 0 - 2 1 6 1 , 3 8}$ & $-\mathbf{x : ~ 3 1 6 , 4 1}$ \\
\hline
\end{tabular}




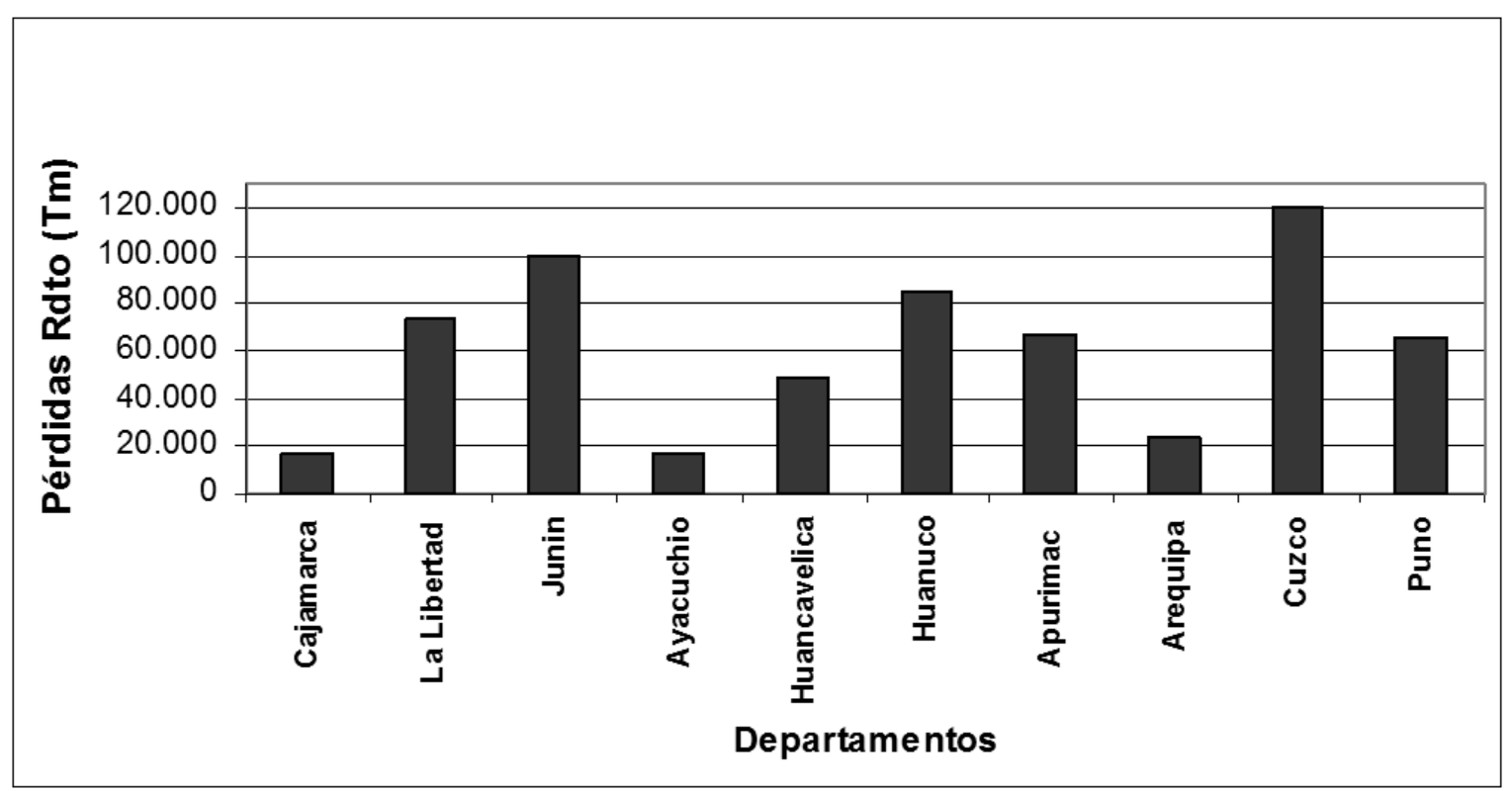

Figura 1. Pérdidas en el rendimiento (t) causadas por Globodera spp. en 10 departamentos Andinos de Perú 
En Bolivia el porcentaje de incidencia fue mayor, aunque el número de muestras fue menor al de Perú (78.42 \% de 735 muestras) y se le encontró infestando mayormente los suelos con poblaciones incipientes $(29.5 \%$ de las comunidades) y medias (25.9\% de las comunidades). Estas infestaciones incipientes ganan mayor importancia en los seis departamentos andinos (35.40\%), afectando mayor número de hectáreas en La Paz, Chuquisaca y Potosí. Seguidamente, las infestaciones medias (21.19\%) y muy altas (18.82\%) tienen similar importancia, es así que las hectáreas afectadas por poblaciones medias, se observan más en los departamentos de La Paz y Potosí y las afectadas por poblaciones muy altas en Cochabamba y La Paz (Figura 2). Las pérdidas en rendimiento estimadas en los cinco departamentos andinos variaron según los niveles de infestación indicados para cada uno de ellos. Se estimaron las mayores pérdidas en los departamentos de $\mathrm{La}$ Paz, Potosí y Chuquisaca, no así en los departamentos de Cochabamba y Tarija (Figura 3).

En general., La Paz, Chuquisaca y Potosí presentaron las mayores pérdidas en el valor bruto de la producción de papa por poblaciones incipientes. Los departamentos que presentaron pérdidas por poblaciones medias y muy altas son La Paz, Potosí y Cochabamba. Por último, La Paz, Potosí y Chuquisaca presentaron las mayores pérdidas por poblaciones altas de Globodera spp.

Los resultados de incidencia y severidad de daño extrapolados con las áreas cultivadas y precio de venta de los tubérculos en Bolivia y Perú han permitido estimar que las pérdidas económicas en el valor bruto de la producción de papa en Bolivia alcanza a US\$13'000,000 y en Perú se estiman en US\$ $128^{`} 000,000$ (Tabla 5 y 6 , respectivamente).

En Perú predomina la especie G. pallida con infestaciones localizadas de $G$. rostochiensis en los departamentos sureños de Arequipa y Puno. En cambio en Bolivia, G. pallida supera ligeramente la incidencia de $G$. rostochiensis. Chuquisaca, La 
Paz y Cochabamba son los más infestados por esta especie, en cambio, Tarija y La Paz por G. rostochiensis. 


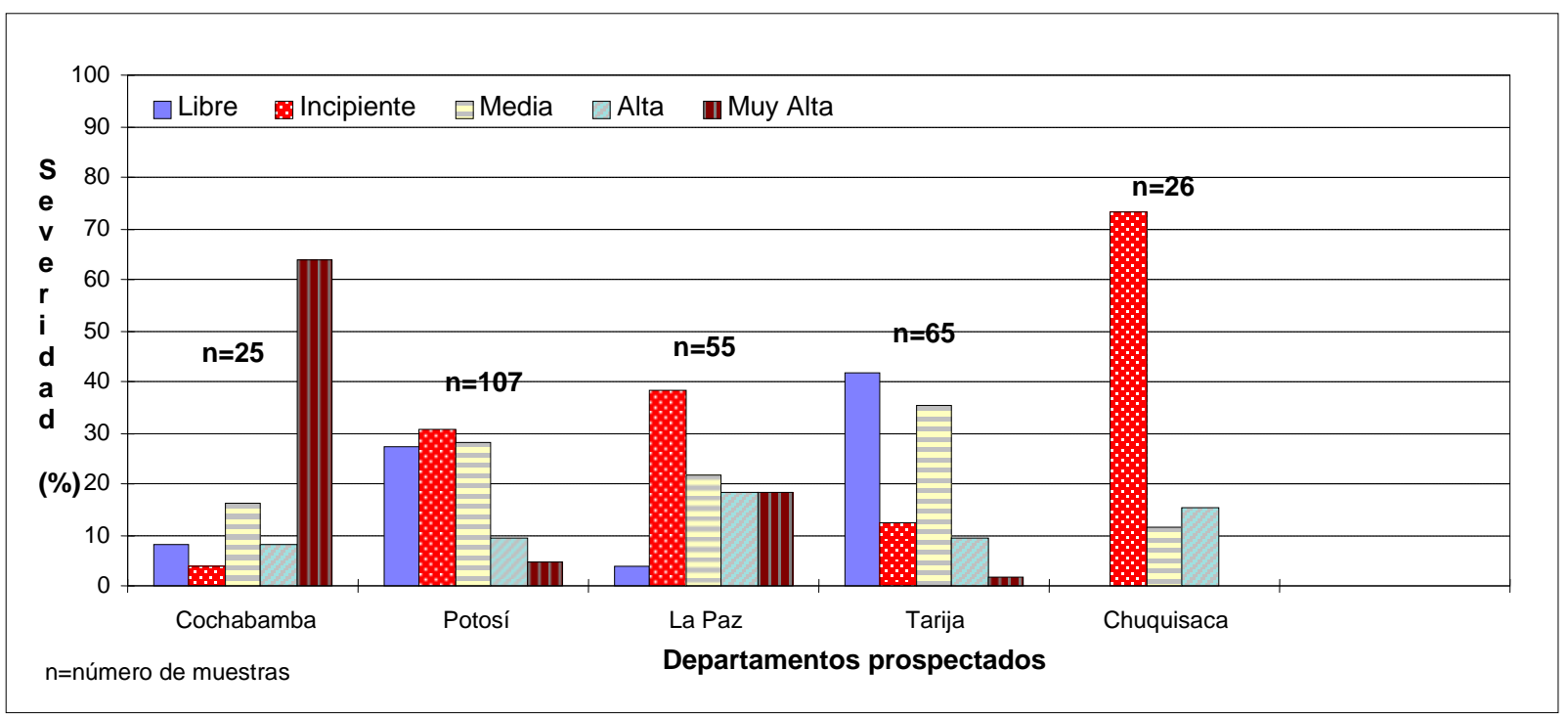

Figura 2. Porcentaje de severidad por efecto de la infestación de los suelos por Globodera spp. en cinco departamentos prospectados en Bolivia 


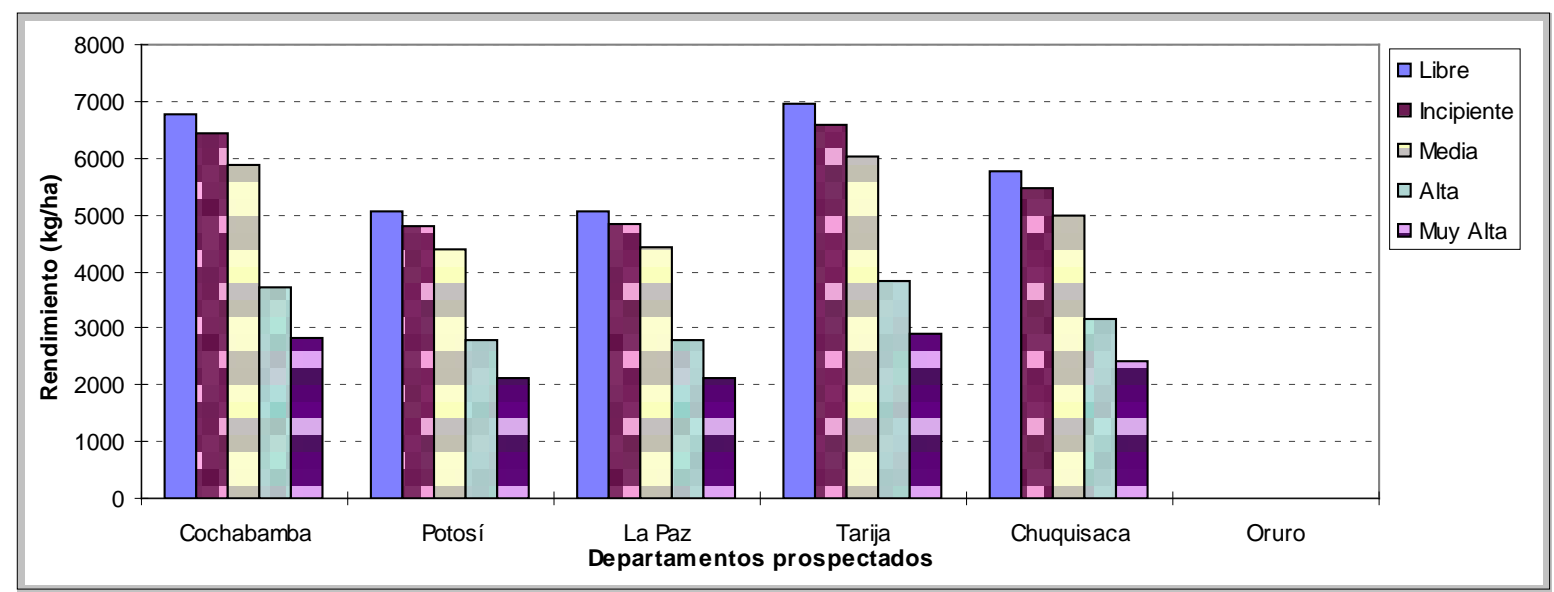

Figura 3. Pérdidas en el rendimiento del cultivo de papa $(\mathrm{kg} / \mathrm{ha})$ ocasionados de acuerdo al grado de severidad de daño causado por Globodera sp. en los diferentes departamentos prospectados en Bolivia 
Por otro lado, Tarija también se encuentra simultáneamente infestada por $G$. pallida y G. rostochiensis. En ambos países la G. pallida y $G$. rostochiensis se encuentran más frecuentemente entre los 3500 a los 4000 msnm.

Los esfuerzos locales aislados, como los efectuados en Bolivia y Perú, deben ser enfocados desde un ángulo regional, porque de otra forma la problemática de Globodera $s p$. se concreta a esfuerzos aislados. En este sentido, es necesario el desarrollo e implementación de una estrategia regional para promover el establecimiento de un proyecto regional que coordine y optimice los avances tecnológicos alcanzados a nivel de cada país.

Tabla 5. Niveles de infestación por Globodera sp. y pérdidas económicas en la región Andina de Perú

\begin{tabular}{|l|c|c|c|}
\hline \multicolumn{1}{|c|}{ Niveles } & $\begin{array}{c}\text { Áreas Infestadas } \\
\text { (has) }\end{array}$ & (\%) & US \$ \\
\hline Libre (0\%) & 75.606 & 34,43 & \\
\hline Incipiente (5\%) & 28.666 & 13,06 & 3.570 .089 \\
\hline Medio (13\%) & 22.913 & 10,44 & 7.569 .055 \\
\hline Alto (45\%) & 23.933 & 10,90 & 27.162 .49 \\
\hline Muy alto (58\%) & 68.448 & 31,17 & 90.091 .61 \\
\hline TOTAL: & $\mathbf{2 1 9 . 5 6 9 ( 1 4 3 , 9 6 3 )}$ & $\mathbf{1 0 0 . 0 ~ ( 6 5 . 5 7 ) ~}$ & $\mathbf{1 2 8 , 3 9 3 , 2}$ \\
\hline
\end{tabular}


Tabla 6. Pérdidas económicas en el valor bruto de la producción (US\$) causadas por la presencia de Globodera sp. en los departamentos andinos de Bolivia

\begin{tabular}{|l|c|}
\hline \multicolumn{1}{|c|}{ Departamento } & US \$ \\
\hline Cochabamba & 6' 278.617 \\
\hline Potosí & 1' 550.637 \\
\hline La Paz & 6' 655.950 \\
\hline Tarija & 387.097 \\
\hline Chuquisaca & 1' 390.515 \\
\hline Total & $\mathbf{1 6} \mathbf{2 6 2 . 8 1 9}$ \\
\hline
\end{tabular}

\section{Agradecimientos}

Se agradece a todos los tesistas que con su esfuerzo y trabajo de campo colaboraron tanto en Universidades de Bolivia y Perú en generar la información sobre las poblaciones del nematodo de quiste Globodera spp. utilizadas en la preparación de este artículo.

\section{Literatura Cítada}

Bell, F. H.; S. Alandia. 1955. Golden nematode and other potato diseases in Bolivia. Plant Disease Reporter 38 (5): 407.

Devaux, A.; M.Ordinola; A. Hibon; R. Flores. 2010. El sector papa en la región Andina: Diagnóstico y elementos para una visión estratégica (Bolivia, Ecuador y Perú). Centro Internacional de la Papa, Lima, Perú. $385 \mathrm{pp}$.

Esprella, R. 1993. Evaluación en parcelas campesinas del nematodo del quiste de la papa (Globodera spp.) en función al tiempo de descanso en el Altiplano Central Boliviano. Tesis Ing. Agr. Facultad de Ciencias Agrícolas y Pecuarias "Martín Cardenas", UMSS. Cochabamba, Bolivia. 99p. 
Evans, K.J.; Franco; M.M. Scurrah. 1975. Distribution of species of potato cyst nematodes in South America. Nematologica 21, 365-369.

Franco, J., 1977. Studies on the taxonomy and biology of potato cyst nematodes Globodera spp. Mulvey and Stone 1976. Thesis, University of London.

Franco, J. 1994. Problemas de nematodos en la producción de papa en climas templados en la región andina. Nematropica 24: 179-195.

Franco, J.; A. Gonzales; A. Matos. 1993. Manejo integrado del nematodo quiste de la papa. Centro Internacional de la Papa (CIP), Programa de Investigación de la Papa (PROINPA), Cochabamba, Bolivia. 172 pp.

Franco, J.; J.Ramos; R. Oros; G. Main; N. Ortuño. 1998/1999. Pérdidas económicas causadas por Nacobbus aberrans y Globodera spp. en el cultivo de la papa en Bolivia. Revista Latinoamericana de la Papa 11: 40-66.

Gonzales, A.; J. Franco. 1993. Técnicas y Métodos para el Nematodo Quiste. Centro Internacional de la Papa y Programa de la Investigación de la Papa. Cochabamba, Bolivia.100 pp.

Hijmans, R.J.; D.M. Spooner. 2001. Geographic distribution of wild potato species. American Journal of Botany 88: 2101-2112.

Huamán, Z; D.M. Spooner. 2002 Reclassification of landrace populations of cultivated potatoes (Solanum sect. Petota). American Journal of Botany 89:947-965.

Instituto Nacional de Estadística (INE); Ministerio de Desarrollo Sostenible y Medio Ambiente. 1994. Boletín de Estadísticas Agrícolas 1992-93-1994. 53p.

Lanza, M. E.1996. Distribución de Nacobbus aberrans y Globodera spp. en zonas paperas de siete provincias del departamento de Potosí. Tesis Ing. Agr. Facultad de Ciencias Agrícolas y Pecuarias, Universidad Autónoma "Tomás Frías". Potosí, Bolivia. 75 p.

Main, G. 1994. Distribución de Globodera spp. en cuatro provincias de La Paz e identificación de fuentes de resistencia. Tesis Ing. Agr. Facultad de Ciencias Agrícolas y Pecuarias, Universidad Técnica de Oruro. Oruro, Bolivia. $94 \mathrm{p}$. 
MINAG. 2007. Mministerio de Agricultura del Perú. Situación de la Cadena Agroproductiva de Papa en Perú. Perú.

Peralta, L. 1995. Distribución e identificación de especies de Globodera spp. en las provincias de Yamparaez y Chayanta. Tesis Ing. Agr. Facultad de Ciencias Agrícolas, Pecuarias y Forestales, Universidad Mayor Real y Pontificia de San Francisco Xavier de Chuquisaca. Sucre, Bolivia.

Tola M., A.. 1997. Diagnóstico de suelos para la detección de Nacobbus aberrans y Globodera spp. en la zona de Araca (Prov. Loayza) La Paz. Tesis Ing. Agr. Facultad de Agronomía, Universidad Mayor de San Andrés. La Paz, Bolivia. 131 p.

Zeballos, H. 1997. Aspectos económicos de la producción de papa en Bolivia. Informe al Centro Internacional de la Papa (CIP) y el Programa de Investigación de la Papa (PROINPA). 209 p.

Wille, J. E.; C. Bazán de Segura, C. 1952. La Anguilula Dorada, Heterodera rostochiensis, una plaga del cultivo de las papas recien descubierta en el Perú. Boletin No. 48, Estación Experimental Agrícola La Molina, Lima, Perú, 17 pp. 